\title{
Riqueza e abundância de morcegos capturados na borda e no interior de um fragmento florestal do estado do Acre, Brasil
}

\author{
Armando Muniz Calouro ${ }^{1 *}$ \\ Francisco Glauco de Araújo Santos ${ }^{2}$ \\ Camila de Lima Faustino ${ }^{1}$ \\ Simey Freitas de Souza ${ }^{1}$ \\ Brenda Moraes Lague ${ }^{1}$ \\ Rodrigo Marciente ${ }^{1}$ \\ Glauco Jonas Lemos Santos ${ }^{3}$ \\ Amanda Oliveira Cunha ${ }^{1}$ \\ ${ }^{1}$ Centro de Ciências Biológicas e da Natureza, Universidade Federal do Acre \\ Rod, BR-364, km 04, CEP 69915-900, Rio Branco-AC, Brasil \\ ${ }^{2}$ Centro de Tecnologia, Departamento de Agropecuária \\ Universidade Federal do Rio Grande do Norte, Natal-RN, Brasil \\ ${ }^{3}$ Faculdade de Medicina Veterinária, Universidade Estadual do Ceará, Fortaleza-CE, Brasil \\ *Autor para correspondência \\ acalouro@bol.com.br
}

Submetido em 22/10/2009

Aceito para publicação em 01/09/2010

\section{Resumo}

Devido ao desmatamento, a fragmentação florestal é uma realidade cada vez mais presente no oeste do estado do Acre. O estudo objetivou avaliar variações na riqueza e abundância de morcegos em um fragmento florestal urbano de aproximadamente 150ha localizado na cidade de Rio Branco (AC). As coletas foram realizadas em dois sítios: sendo dois pontos de coleta a 200m da borda e outros dois a $20 \mathrm{~m}$ da borda. Redes de neblina ficaram abertas quatro horas depois do pôr-do-sol, durante três noites/mês. O índice de diversidade de ShannonWiener $\left(\mathrm{H}^{\prime}\right)$ e o índice de similaridade de Jaccard $(\mathrm{J})$ foram usados para análise comparativa. Durante 48 noites (novembro/2005-julho/2007) foram capturados 85 morcegos de 15 espécies, com maior riqueza e abundância de quirópteros no interior do fragmento. A similaridade de espécies foi baixa $(\mathrm{J}=0,44)$, indicando preferência de habitat. $\mathrm{O}$ índice de diversidade encontrado $\left(\mathrm{H}^{\prime}=2,091\right.$ nats/indiv) foi similar a de outros estudos na Amazônia, com menor diversidade na borda $\left(\mathrm{H}^{\prime}=1,864\right)$ do que no interior do fragmento $\left(\mathrm{H}^{\prime}=2,047\right)$. Carollia perspicillata $(\mathrm{n}=32)$ e Artibeus lituratus $(\mathrm{n}=13)$ responderam por 57,6\% do total de capturas. As características da matriz e a adaptação dos morcegos às redes explicam os valores encontrados.

Unitermos: Acre, Amazônia, preferência de habitat, quirópteros, riqueza 


\section{Abstract}

Richness and abundance of bats captured at the edge and within a forest fragment in Acre, Brazil. As a consequence of deforestation, forest fragmentation is a reality that is increasingly present in the western part of the state of Acre, Brazil. The objective of this study was to evaluate variations in the richness and abundance of bats in an urban forest fragment of approximately 150ha, which is located in Rio Branco (AC). Collections were performed in two places: two points that were $200 \mathrm{~m}$ from the edge and two that were $20 \mathrm{~m}$ from the edge. Mist-nets were opened four hours after sundown during three nights/month. The Shannon-Wiener diversity index $\left(H^{\prime}\right)$ and the Jaccard Similarity index $(\mathrm{J})$ were used for comparative analysis. On 48 nights (November/2005 to July/2007), 85 individual bats of 15 species were captured, with greater richness and abundance of chiropters within the fragment. The similarity index of species was low $(\mathrm{J}=0.44)$, indicating habitat preference. The diversity index $\left(\mathrm{H}^{\prime}=2.091\right)$ was similar to that of previous studies in the Amazon, especially in Brazil, with lower diversity at the edge $\left(\mathrm{H}^{\prime}=1.864\right)$ than inside the fragment $\left(\mathrm{H}^{\prime}=2.047\right)$. Carollia perspicillata $(\mathrm{n}=32)$ and Artibeus lituratus $(n=13)$ were responsible for $57.6 \%$ of the total captured. The characteristics of the matrix and the adaptation of the bats to the mist-nets explained the observed values.

Key words: Acre, Amazon, bats, habitat preference, richness

\section{Introdução}

A única estimativa da riqueza de quirópteros no estado do Acre foi realizada por Nogueira et al. (1999), os quais estimaram a ocorrência de 57 espécies de morcegos no Estado. Entretanto, esse número deve estar subestimado, pois existem somente duas referências bibliográficas específicas sobre coleta de quirópteros no Acre: uma realizada no Parque Nacional da Serra do Divisor (Nogueira et al., 1999) e outra nas cercanias da cidade de Cruzeiro do Sul (Taddei et al., 1990), ambas no Vale do Juruá, oeste do Estado. Assim, o grupo permanece praticamente desconhecido na parte leste do Estado, como por exemplo, ao redor da capital, Rio Branco. Essa região está em um processo crescente de fragmentação florestal (Acre, 2006), com as populações de animais e plantas sofrendo os efeitos deletérios inerentes a esse tipo de perturbação antrópica, como o isolamento genético e o efeito de borda.

O efeito de borda é fruto das mudanças nos padrões de incidência de luz, vento, temperatura e umidade na borda do fragmento, com reflexos na composição florística e, consequentemente, na comunidade de animais (Laurance e Bierregaard, 1997). No caso dos morcegos, a composição de espécies em uma área está relacionada, principalmente, com a disponibilidade de abrigo, alimento e a estrutura da vegetação (Kunz e Fenton, 2003). Assim, variações na composição e na estrutura vegetacional na borda do fragmento tenderiam a alterar a ocorrência de espécies de morcegos nesses locais. De modo geral, esse grupo taxonômico é considerado um bom indicador de qualidade ambiental por apresentar alta diversidade de espécies e de nichos ecológicos, refletindo desta forma, variações ambientais existentes entre a borda e o interior de um fragmento florestal (Fenton et al., 1992; Medellín et al., 2000; Henry et al., 2007).

O efeito da fragmentação florestal sobre os quirópteros foi pouco estudado no Brasil. A maior parte dos estudos se concentra no sul e sudeste do país, com enfoque na riqueza e a abundância de morcegos existentes nos fragmentos e como essas variáveis estão relacionadas com o tamanho da área e as características ambientais do fragmento e da matriz circundante (Pedro et al., 1995; Reis e Muller, 1995; Reis et al., 2000; 2003; Félix et al., 2001; Barros et al., 2006; Carvalho et al., 2009). Outras abordagens ecológicas também procuram avaliar a capacidade das espécies em superar as distâncias existentes entre os fragmentos (Bianconi et al., 2006; Costa et al., 2006; Menezes et al., 2008), além de medir variações nas diversidades de morcegos que utilizam a borda e o interior de fragmentos de diferentes tamanhos (Faria, 2006).

Estudos amazônicos relacionando fragmentação e diversidade de quirópteros são escassos e pontuais, tendo sido realizados na Guiana Francesa (Cosson et al., 1999; Henry et al., 2007), em Iquitos, Peru (Willig et al., 2007; Klingbeil e Willig, 2009) e, no Brasil, nos estados do Pará e Amazonas (Bernard e Fenton, 2002; 
2003; 2007; Sampaio et al., 2003). Os resultados não apontam para uma resposta padrão à fragmentação, mas ao tamanho do fragmento, a sua integridade ambiental e, principalmente, as características da matriz circundante que parecem ser determinantes nas variações encontradas na riqueza e abundância das espécies.

Este estudo teve o objetivo de determinar a riqueza de espécies de morcegos e abundância de indivíduos capturados na borda e no interior de um fragmento florestal urbano existente na cidade de Rio Branco (AC).

\section{Material e Métodos}

\section{Área de estudo}

O Parque Zoobotânico (PZ), com uma área de cerca de 150ha (Figura 1), é um fragmento florestal urbano situado no Campus Universitário da Universidade Federal do Acre (UFAC), no município de Rio Branco, Acre (9'57'26"S e 6752'25'W). Segundo a classificação de Köppen, o PZ apresenta um clima tropical úmido, com a estação seca compreendendo o período de maio a outubro, sendo junho o mês menos chuvoso. A vegetação do $\mathrm{PZ}$ é formada por florestas em diferentes estágios sucessionais, gerados pelas mortandades simultâneas de maciços de bambus nativos, especialmente Guadua weberbaueri, com mortandade simultânea registrada a cada 32 anos (Silveira, 2005). Segundo Meneses-Filho et al. (1995), a área onde se encontra o PZ fazia parte na década de 1940 de um seringal conhecido como "Empresa" e as atividades antrópicas eram basicamente extrativistas. A área não possui registro histórico de retirada de madeira ou fogo desde a inauguração do campus em 1976. Ainda segundo Meneses-Filho et al. (1995), os tipos florestais existentes no PZ podem se apresentar em mosaicos com transições pouco perceptíveis na estrutura da vegetação e composição florística, formando contínuos vegetacionais com sub-bosque denso. De maneira geral, a dinâmica populacional do bambu gerou três tipos básicos de formação vegetal no PZ: floresta ombrófila aberta de palmeiras, floresta ombrófila aberta de palmeiras com bambu dominado e floresta ombrófila aberta com bambu dominante (conhecido localmente como tabocal). O tabocal é uma tipologia florestal natural da região, porém sua ocorrência e dispersão em uma área podem ser beneficiadas pela atividade antrópica, já que o bambu Guadua weberbaueri cresce rapidamente em condições de maior luminosidade. No caso do PZ, que é um fragmento florestal urbano, esse tipo de vegetação ocorre principalmente nas áreas que estão sob efeito de borda (com maior luminosidade), e por ser uma espécie competitivamente eficiente (rápido crescimento e inibidora da regeneração de outras espécies), domina a paisagem no $\mathrm{PZ}$ até $100 \mathrm{~m}$ a partir da borda do fragmento. O tabocal também ocorre no interior do fragmento, mas em pequenas manchas de cerca de 30 a $40 \mathrm{~m}$ de raio (observações pessoais). Devido à dinâmica natural do bambu Guadua weberbaueri (mortandade simultânea a cada 32 anos) existe um mosaico de vegetação no PZ que é consequência desse fenômeno, o que permite considerar toda a área como Floresta Secundária [ver Silveira (1995) para maior detalhe sobre a biologia da espécie Guadua weberbaueri].

Dois pontos de coleta (Pontos 1 e 3 ) foram situados a $200 \mathrm{~m}$ da borda, em floresta aberta com palmeiras, sem a presença de bambu. Outros dois pontos de coleta (Pontos 2 e 4) foram localizados a 20m da borda oeste do PZ, fazendo divisa com o Campus Universitário, composta por floresta aberta de palmeiras com bambu dominado. Em cada ponto de coleta foram abertos dois transectos, onde foram colocadas as redes de neblina de até $3 \mathrm{~m}$ de altura, enfocando a captura de morcegos que utilizam o sub-bosque (Figura 1).

\section{Capturas e análise dos dados}

Os morcegos foram capturados com o auxílio de redes de neblina, abertas no mesmo horário, ao longo de quatro horas por noite, depois do pôr-do-sol (Kunz et al., 1996). Nos Pontos 1 e 2, os morcegos foram capturados com redes de $7 \mathrm{~m} \times 2,5 \mathrm{~m}$ e malha de $38 \mathrm{~mm}$, sendo colocadas dez redes em cada ponto de coleta (cinco redes em cada transecto), totalizando $350 \mathrm{~m}^{2}$ de rede por noite. Foram realizadas 12 coletas (três noites em cada coleta) de novembro de 2005 a agosto de 2006, totalizando 36 noites de coleta. Assim, tanto no Ponto 1 como no Ponto 2, foi realizado um esforço de coleta de $25.200 \mathrm{~m}^{2}$.h $\left(17,5 \mathrm{~m}^{2} \times 4 \mathrm{~h} \times 36\right.$ noites $\times 10$ redes $)$, 


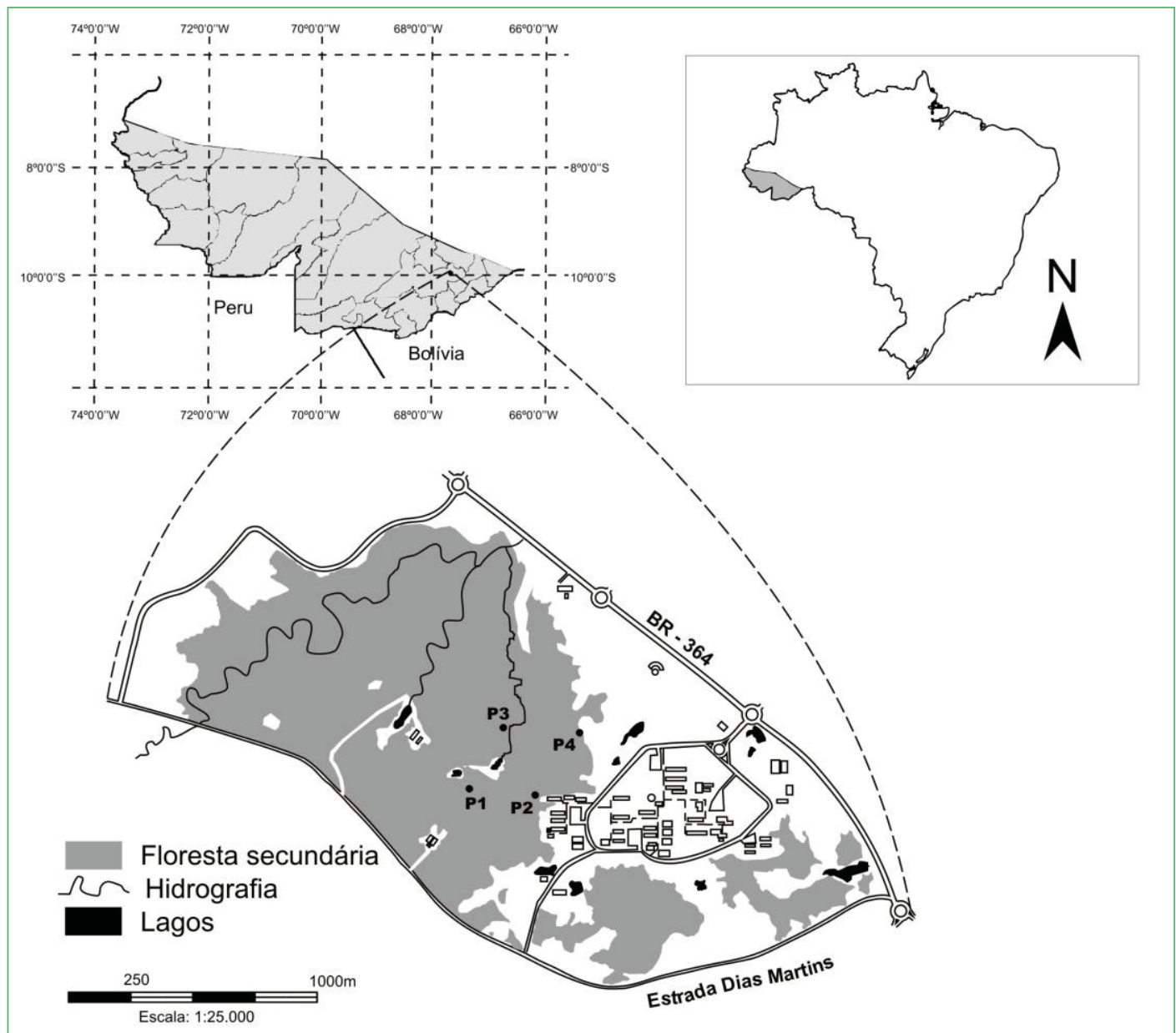

FIGURA 1: Localização da área de estudo (PZ/UFAC) com a disposição dos pontos de coleta do interior do fragmento florestal (Pontos 1 e 3) e os da borda (Pontos 2 e 4).

conforme Straube e Bianconi (2002). Já nos Pontos 3 e 4 foram usadas seis redes de neblina de $12 \mathrm{~m} \times 2,5 \mathrm{~m}$ e malha de $38 \mathrm{~mm}$ em cada ponto de coleta (três em cada transecto), totalizando $360 \mathrm{~m}^{2}$ de rede por noite. Foram realizadas quatro coletas (três noites em cada coleta) no período de março a julho de 2007, totalizando 12 noites de coleta. Tanto no Ponto 3 como no Ponto 4 foi realizado um esforço de $8.640 \mathrm{~m}^{2}$.h. Assim, o esforço de captura realizado no $\mathrm{PZ}$ totalizou $67.680 \mathrm{~m}^{2}$.h, dividido igualitariamente na borda e no interior do fragmento.

Não foram realizadas coletas em noites com chuvas ou com friagem (caracterizadas por queda abrupta de temperatura), focando as noites próximas da lua nova para evitar efeitos de fobia lunar e maximizar as taxas de captura (Esbérard, 2007). Depois de abertas, as redes foram checadas a cada $20 \mathrm{~min}$ e os animais capturados foram pesados. No máximo três exemplares de cada espécie foram eutanaziados e incorporados à Coleção Zoológica de Mamíferos da UFAC. Os morcegos foram identificados com o auxílio das descrições realizadas por Vizzoto e Taddei (1973), Marques-Aguiar (1994) e Eisenberg e Redford (1999). A nomenclatura taxonômica adotada foi a proposta por Wilson e Reeder (2005). A análise da diversidade de morcegos nos dois tratamentos (borda e interior) foi feita através do índice de diversidade de Shannon-Wiener (H') e do índice de similaridade de Jaccard (J), descritos em Ludwig e Reynolds (1988). O índice de similaridade de Jaccard mostra a semelhança entre as duas comunidades em termos de composição de espécies, variando de zero (diferença máxima) a um (idênticas). Para estimativa da riqueza existente no $\mathrm{PZ}$ foi utilizado o método nãoparamétrico Jackknife 2 (segunda ordem), descrito em 
Santos (2003). Tanto o índice de similaridade como a estimativa de riqueza foram calculados com o auxílio do Programa Biodiversity Pro 4.0. Para testar diferenças entre as abundâncias encontradas na borda e no interior do fragmento foi utilizado um teste $t$, aplicado através do programa PAST (Hammer et al., 2001), o qual também foi utilizado para calcular e comparar os índices de diversidade, com uso de logaritmo neperiano em sua fórmula.

\section{Resultados}

No total, foram capturados 85 morcegos de 15 espécies no Parque Zoobotânico da UFAC (Tabela 1). Dentre eles, destacam-se Carollia perspicillata $(\mathrm{n}=$ 32) e Artibeus litutarus ( $\mathrm{n}=13$ ), ambas as espécies frugívoras. No interior do fragmento foi registrada a maior riqueza de morcegos ( $n=14$ espécies $)$ e o maior número de capturas $(\mathrm{n}=61)$, em comparação com o menor número de espécies $(\mathrm{n}=8)$ e de capturas $(\mathrm{n}=24)$ encontrada na borda. Estatisticamente, essa diferença no número de capturas foi considerada significativa ( $t$ $=2,209, \mathrm{p}=0,04431$ ).

A curva cumulativa de espécies (Figura 2) indica que com o aumento do esforço amostral provavelmente exista um incremento na riqueza já que a curva ainda não atingiu a assintota. Pelo método Jackknife 2 a estimativa da riqueza esperada de morcegos no PZ é de 26,87 espécies. Assim, foram capturadas 55,8\% das espécies existentes no PZ com um esforço de $67.680 \mathrm{~m}^{2}$.h. Já a estimativa de riqueza esperada para o interior do $\mathrm{PZ}$ foi de 23,13 espécies (com a captura real de 60,5\% do esperado) e de 9,98 espécies para a borda do fragmento (com captura real de $80,1 \%$ do esperado).

O índice de diversidade de Shannon-Wiener, encontrado no $\mathrm{PZ}$, foi de $\mathrm{H}^{\prime}=2,091$ e a comparação

TABELA 1: Espécies de morcegos capturadas em quatro pontos de coleta (P) no Parque Zoobotânico da UFAC: (I - 200m da borda; B - 20m da borda).

\begin{tabular}{|c|c|c|c|c|c|c|c|}
\hline Espécie & P1(I) & P2(B) & P3(I) & P4(B) & I & $\mathbf{B}$ & Total \\
\hline \multicolumn{8}{|l|}{ PHYLLOSTOMIDAE } \\
\hline \multicolumn{8}{|l|}{ Subfamília Carollinae } \\
\hline Carollia perspicillata (Linnaeus, 1758) & 19 & 5 & 5 & 3 & 24 & 8 & 32 \\
\hline Rhynophylla pumilio Peters, 1865 & 0 & 0 & 0 & 1 & 0 & 1 & 1 \\
\hline \multicolumn{8}{|l|}{ Subfamília Stenodermatinae } \\
\hline Artibeus cinereus (Gervais, 1856) & 2 & 1 & 2 & 1 & 4 & 2 & 6 \\
\hline Artibeus lituratus Olfers, 1818 & 10 & 3 & 0 & 0 & 10 & 3 & 13 \\
\hline Artibeus planirostris Spix, 1823 & 5 & 0 & 0 & 0 & 5 & $\mathbf{0}$ & 5 \\
\hline Platyrrhinus helleri (Peters, 1866) & 1 & 3 & 1 & 1 & 2 & 4 & 6 \\
\hline \multicolumn{8}{|l|}{ Subfamília Glossophaginae } \\
\hline Lonchophylla tomasi J. A. Allen, 1904 & 1 & 0 & 0 & 0 & 1 & $\mathbf{0}$ & 1 \\
\hline \multicolumn{8}{|l|}{ Subfamília Phyllostominae } \\
\hline Lophostoma silvicolum d'Orbigny, 1836 & 1 & 0 & 0 & 0 & 1 & $\mathbf{0}$ & 1 \\
\hline Mimon crenulatum (E. Geoffroy, 1803) & 1 & 0 & 0 & 0 & 1 & $\mathbf{0}$ & 1 \\
\hline Mycronycteris minuta (Gervais, 1856) & 0 & 0 & 2 & 0 & 2 & $\mathbf{0}$ & 2 \\
\hline Phyllostomus elongatus (E. Geoffroy, 1810) & 3 & 1 & 1 & 2 & 4 & 3 & 7 \\
\hline Phyllostomus hastatus (Pallas, 1767) & 3 & 0 & 0 & 1 & 3 & 1 & 4 \\
\hline Tonatia saurophila Koopman and Williams, 1951 & 2 & 0 & 0 & 2 & 2 & 2 & 4 \\
\hline \multicolumn{8}{|l|}{ VESPERTILIONIDAE } \\
\hline \multicolumn{8}{|l|}{ Subfamília Vespertilioninae } \\
\hline Eptesicus brasiliensis (Desmarest, 1819) & 0 & 0 & 1 & 0 & 1 & $\mathbf{0}$ & 1 \\
\hline Eptesicus sp. & 0 & 0 & 1 & 0 & 1 & $\mathbf{0}$ & 1 \\
\hline Total de espécies & 11 & 5 & 7 & 7 & 14 & 8 & 15 \\
\hline Total de indivíduos & 48 & 13 & 13 & 11 & 61 & 24 & 85 \\
\hline
\end{tabular}




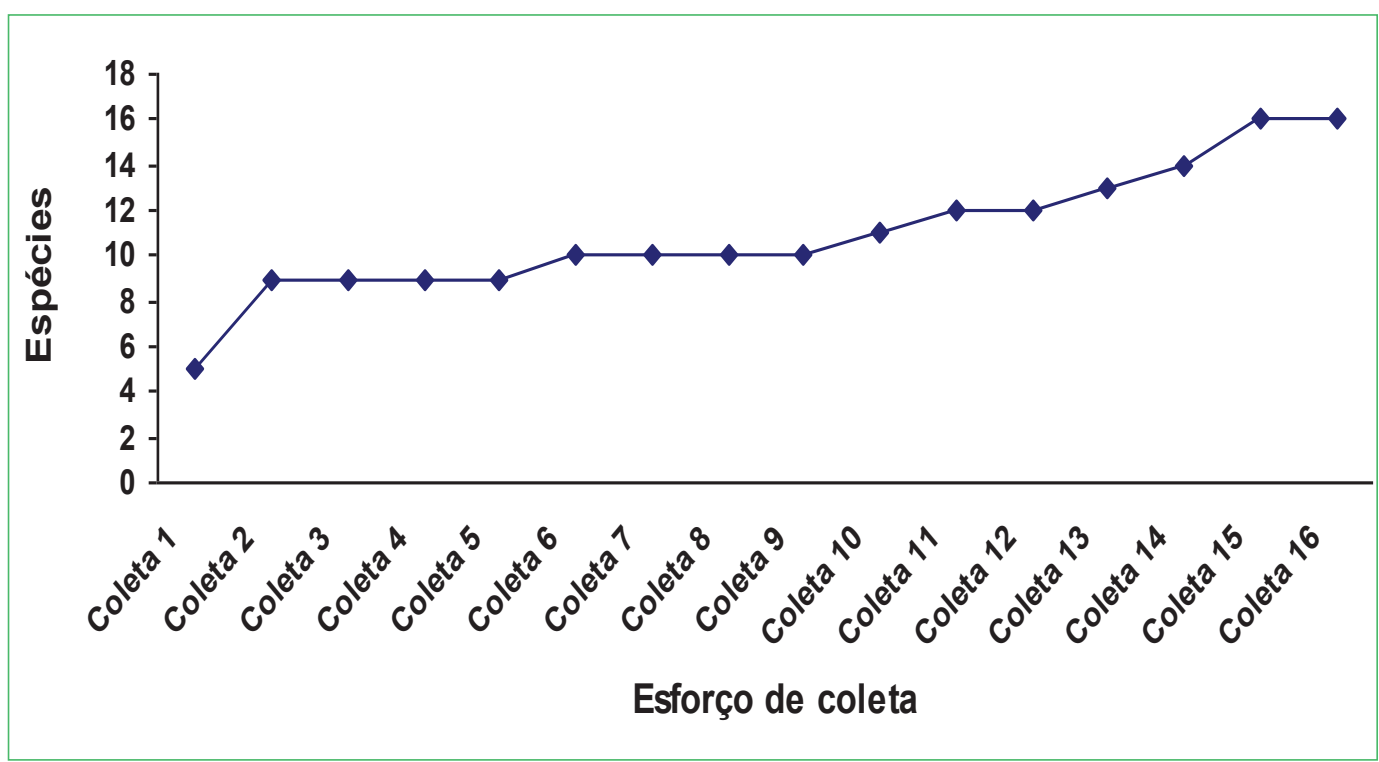

FIGURA 2: Curva cumulativa de espécies de quirópteros registradas a cada esforço de coleta no Parque Zoobotânico: coletas 1 a 12 realizadas nos Pontos 1 e 2 (novembro de 2005 a agosto de 2006), com esforço de $4.200 \mathrm{~m}^{2}$.h a cada coleta; coletas 13 a 16 realizadas nos Pontos 3 e 4 (março a julho de 2007), com esforço de $4.320 \mathrm{~m}^{2}$.h a cada coleta.

do índice do interior $\left(\mathrm{H}^{\prime}=2,047\right)$ com o encontrado na borda $\left(H^{\prime}=1,864\right)$ indicou que não existe uma diferença significativa nítida entre eles $(\mathrm{p}=0,516)$.

O número de espécies registradas no interior do fragmento $(\mathrm{n}=14)$ foi maior do que o registrado na borda $(\mathrm{n}=8)$, conforme observado na Tabela 1 , mas a similaridade em termos de composição de espécies não foi alta $(J=0,44)$. Entretanto, a predominância de Carollia perspicillata e Artibeus lituratus no número de registros foi maior no interior, já que essas duas espécies responderam por $57,6 \%$ do total de indivíduos capturados no interior do fragmento, contra $33,3 \%$ na borda (Tabela 1).

\section{Discussão}

O número de animais capturados $(\mathrm{n}=85)$ foi baixo quando consideramos o esforço amostral empregado. Segundo Sampaio et al. (2003), na região Neotropical são necessárias cerca de 1.000 horas-rede para capturar cerca de $80 \%$ das espécies de morcegos da área pesquisada, mas o método Jackniffe 2 indicou que somente $55,8 \%$ da riqueza esperada foi capturada no PZ (em um esforço equivalente a 1.728 horas-rede). A adaptação dos morcegos às redes pode ter sido uma das causas, pois segundo Esbérard (2006), ocorre uma queda significativa na taxa de captura quando as coletas são realizadas nos mesmos locais, mas com uma diminuição não significativa entre noites subsequentes de coleta.

A dominância observada para Carollia e Artibeus no PZ encontra amparo na literatura (Reis, 1984; Pedro et al., 1995; Reis et al., 2000; Bernard, 2002; Bianconi et al., 2004). O método de captura com rede de neblina até a altura de $3 \mathrm{~m}$ tem o viés de coletar predominantemente frugívoros filostomídeos que utilizam o sub-bosque, como é o caso desses dois gêneros. Além disso, Carollia e Artibeus se adaptam bem a ambientes perturbados, pois consomem frutos de espécies vegetais pioneiras presentes no sub-bosque (Faria, 2006), de forma mais ampla no caso de Carollia (Bernard, 2001, 2002) ou de forma mais oportunista, quando ocorre uma escassez de frutos nas copas, no caso de Artibeus (Bonaccorso e Gush, 1987; Bernard, 2001; Kalko e Handley, 2001).

Não foi constatada uma diferença significativa entre os índices de diversidade nos dois ambientes, mas foi observada uma diferença significativa entre as abundâncias registradas no interior e na borda. Características diferentes de estrutura de vegetação, provocadas pelo bambu, parecem ser a causa dessa variação, que refletiu também na riqueza de espécies e na similaridade das duas comunidades analisadas. 
A baixa similaridade encontrada entre as espécies capturadas na borda e no interior indica que os dois habitats possuem características ambientais que atraem diferentes espécies de morcegos, já que todas as espécies possuem capacidade de deslocamento medida em quilômetros, que abrangem a borda e o interior (Cosson et al., 1999; Bernard e Fenton, 2003) Essa preferência de habitat fica evidenciada pelo fato de que sete espécies de morcegos foram registradas somente no interior do fragmento contra uma espécie registrada somente na borda (Tabela 1). Entretanto, duas das espécies encontradas no interior do fragmento são insetívoros da Família Vespertilionidae, que também ocorrem em áreas abertas, o que denota que este ambiente tem características de estrutura de vegetação (sub-bosque menos denso) que possibilita a ocorrência de mais espécies, como fica evidenciado pela estimativa de riqueza esperada no interior (cerca de 60\%) ser bem menor do que a estimativa obtida na borda (cerca de $80 \%$ ).

Apesar de não terem sido realizados estudos sobre a composição florística e estrutura da vegetação nos pontos de coleta, algo pode ser inferido pela ocorrência do bambu. Segundo Silveira (2005), o bambu Guadua weberbaueri tem rápido crescimento, principalmente em bordas de fragmentos e clareiras, e tende a diminuir a composição florística da área em que ocorre através da competição por luz e nutrientes com as plântulas. Além disso, essa é uma espécie escandente, o que torna o sub-bosque denso. A menor variedade de fontes alimentares vegetais, menor variedade de abrigos, além da maior dificuldade de vôo no sub-bosque denso são as prováveis causas da menor riqueza e abundância de morcegos registrada no sub-bosque da borda. Se esses padrões de diversidade se mantêm nos estratos superiores da floresta, acima do bambu, é uma hipótese ainda a ser testada.

A diversidade de morcegos do PZ $\left(\mathrm{H}^{\prime}=2,091\right)$ se enquadrou dentro dos valores encontrados no estudo de Klingbeil e Willig (2009) realizado em fragmentos florestais na Amazônia Peruana (variação de 1,93 a 2,89). Segundo Willig et al. (2007), em estudo realizado na mesma região, essas variações nos índices de diversidade estão relacionadas com as paisagens perturbadas em que estão inseridos os fragmentos, pois estas parecem afetar mais a abundância do que a riqueza de espécies de morcegos, desde que exista um mosaico de ambientes com conectividade para os animais se deslocarem. No caso dos morcegos do PZ, os efeitos da perturbação natural gerada pela dinâmica populacional do bambu possivelmente são compensados pela permeabilidade da matriz circundante.

Muitas espécies de morcegos adentram as áreas abertas e são capazes de voar de um fragmento florestal para outro, evitando o isolamento e ampliando a área de forrageio. Mas, a distância que o fragmento se encontra de outras áreas florestais e o tipo de matriz circundante são fatores que podem limitar a riqueza de espécies. Bernard e Fenton (2003), em estudo realizado no Pará sobre a capacidade de morcegos amazônicos se deslocarem entre fragmentos florestais cercados por vegetação de cerrado, encontraram maior capacidade em Carollia perspicillata (até 2,5km de deslocamento), em Artibeus cinereus $(1,9 \mathrm{~km})$ e nenhuma para Mimon crenulatum. Entretanto, os autores não encontraram uma relação entre o peso do corpo e a mobilidade das espécies, já que a matriz permeável formada pela vegetação de cerrado influenciou na capacidade de algumas espécies se deslocarem entre as áreas. Cosson et al. (1999), por outro lado, em estudo realizado em ilhas formadas em um lago de hidrelétrica na Guiana Francesa, demonstraram que somente espécies de maior porte e envergadura como as espécies de Artibeus possuem a capacidade de vôo necessária para atravessar uma matriz inóspita e forragear no maior número de ilhas. Espécies menores forrageiam somente nas ilhas em que foram isoladas (caso de Rhynophilla pumilio), enfrentando os aspectos populacionais negativos oriundos da restrição de área (aumento da competição e endogamia). Desse modo, o grau de permeabilidade da matriz em uma paisagem alterada parece ser um fator mais impactante na riqueza de espécies de morcegos do que os efeitos gerados pela fragmentação.

O PZ é cercado pelo Campus da UFAC, por conjuntos habitacionais e áreas abertas (campos) que estão em processo de urbanização. Fragmentos florestais maiores estão a cerca de dois quilômetros de distância, mas existem dois fragmentos de matas ciliares com menos de 5ha entre o PZ e esses locais, mas que não chegam a formar corredores de vegetação. 
Assim, baseado nos estudos citados, várias espécies de morcegos possivelmente conseguem utilizar essa matriz, explicando a riqueza de espécies encontradas em um fragmento como o $\mathrm{PZ}$, onde há predomínio de florestas secundárias naturais geradas pela dinâmica populacional do bambu. Entretanto, estudos de captura e recaptura de morcegos na matriz e nos fragmentos próximos são necessários para demonstrar até que ponto a riqueza de morcegos existente no PZ é fruto da permeabilidade da matriz circundante.

\section{Agradecimentos}

Ao Fundo de Ciência e Tecnologia do Estado do Acre (FDCT) por financiar o projeto de pesquisa. Ao apoio do CNPq, via fornecimento de bolsas de iniciação científica para alunos de graduação. À direção do Parque Zoobotânico da UFAC por autorizar a realização do estudo.

\section{Referências}

Acre - Governo do Estado do Acre. 2006. Programa Estadual de Zoneamento Ecológico-Econômico do Estado do Acre, Fase II: Documento síntese - Escala 1:250.000. Secretaria Estadual de Meio Ambiente, Rio Branco, Brasil, 334pp.

Barros, R. S. M.; Bisaggio, E. L.; Borges, R. C. 2006. Morcegos (Mammalia, Chiroptera) em fragmentos florestais urbanos no município de Juiz de Fora, Minas Gerais, sudeste do Brasil. Biota Neotropica, 6 (1): versão eletrônica.

Bernard, E. 2001. Vertical stratification of bat communities in primary forest of Central Amazon, Brazil. Journal of Tropical Ecology, 17: 115-126.

Bernard, E. 2002. Diet, activity and reproduction of bats species (Mammalia, Chiroptera) in Central Amazonia, Brazil. Revista Brasileira de Zoologia, 19 (1): 173-188.

Bernard, E.; Fenton, M. B. 2002. Species diversity of bats (Mammalia, Chiroptera) in forest fragments, primary forests and savannas in central Amazonia, Brazil. Canadian Journal of Zoology, 80: 11241140 .

Bernard, E.; Fenton, M. B. 2003. Bat mobility and roosts in a fragmented landscape in central Amazonia, Brazil. Biotropica, 35 (2): 262-277.

Bernard, E.; Fenton, M. B. 2007. Bats in a fragmented landscape: species composition, diversity and habit interactions in savannas of Santarém, Central Amazonia, Brazil. Biological Conservation, 134: 332-343.

Bianconi, G. V.; Mikich, S. B.; Pedro, W. A. 2004. Diversidade de morcegos (Mammalia, Chiroptera) em remanescentes florestais do município de Fênix, noroeste do Paraná, Brasil. Revista Brasileira de Zoologia, 21 (4): 943-954.

Bianconi, G. V.; Mikich, S. B.; Pedro, W. A. 2006. Movement of bats (Mammalia, Chiroptera) in Atlantic Forest remnants in southern Brazil. Revista Brasileira de Zoologia, 23 (4): 1199-1206.

Bonaccorso, F. J.; Gush, T. J. 1987. Feeding behaviour and foraging strategies of captive phyllostomid fruit bats: an experimental study. The Journal of Animal Ecology, 56 (3): 907-920.

Carvalho, F.; Zocche, J. J.; Mendonça, R. Á. 2009. Morcegos (Mammalia, Chiroptera) em restinga no município de Jaguaruna, sul de Santa Catarina, Brasil. Biotemas, 22 (3): 193-201.

Cosson, J. F.; Pons, J. M.; Masson, D. 1999. Effects of forest fragmentation on frugivorous and nectarivorous bats in French Guiana. Journal of Tropical Ecology, 15: 515-534.

Costa, L. M.; Prata, A. F. D.; Moraes, D.; Conde, C. F. V.; JordãoNogueira, T.; Ésberard, C. E. L. 2006. Deslocamento de Artibeus fimbriatus sobre o mar. Chiroptera Neotropical, 12 (2): 289-290.

Eisenberg, J. F.; Redford, K. H. 1999. Mammals of the Neotropics. v 3. The University of Chicago Press, Chicago, USA, 609pp.

Esbérard, C. E. L. 2006. Efeito da coleta de morcegos por noites seguidas no mesmo local. Revista Brasileira de Zoologia, 23 (4): 1093-1096.

Esbérard, C. E. L. 2007. Influência do ciclo lunar na captura de morcegos Phyllostomidae. Iheringia Série Zoologia, 97 (1): 81-85.

Faria, D. 2006. Phyllostomid bats of a fragmented landscape in the nort-eastern Atlantic forest, Brazil. Journal of Tropical Ecology, 22: 531-542.

Félix, J. S.; Reis, N. R.; Lima, I. P.; Costa, É. F.; Peracchi, A. L. 2001. Is the area the Arthur Thomas Park, with its $82.72 \mathrm{ha}$, sufficient to maintain viable chiropteran population? Chiroptera Neotropical, 7 (1-2): 129-133

Fenton, M. B.; Acharya, L.; Audent, D.; Hickey, M. B. C.; Merriman, C.; Obrist, M. K.; Syme, D. M.; Adkins, B. 1992. Phyllostomid bats (Chiroptera: Phyllostomidae) as indicators of habitat disruption in the Neotropics. Biotropica, 24 (3): 440-446.

Hammer, O.; Harper, D. A. T.; Ryan, P. D. 2001. PAST: Paleontological statistics software package for education and data analysis. Palaeontologia Electronica, 4 (1): 1-9.

Henry, M.; Cosson, J. F.; Pons, J. M. 2007. Abundance may be a misleading indicator of fragmentation-sensitivity: The case of figeating bats. Biological Conservation, 139: 462-467.

Kalko, E. K. V.; Handley, C. O. 2001. Neotropical bats in the canopy: Diversity community structure, and implications for conservation. Plant Ecology, 153: 319-333.

Klingbeil, B. T.; Willig, M. R. 2009. Guild-specific responses of bats to landscape composition and configuration in fragmented Amazonian rainforest. Journal of Applied Ecology, 46: 203-213.

Kunz, T. H.; Fenton, M. B. 2003. Bat Ecology. The University of Chicago Press, Chicago, USA, 779pp.

Kunz, T. H.; Thomas, D. W.; Richards, G. C.; Tidemann, C. R.; Pierson, E. D.; Racey, P. A. 1996. Observational techniques for bats. In: Wilson, D. E.; Cole, F. R.; Nichols, J. D.; Rudran, R. F. \& Foster, M. S. (Eds). Measuring and Monitoring Biological Diversity: Standard methods for mammals. Smithsonian Institution Press, Washington, USA, p.105-114. 
Laurance, W. F.; Bierregaard, R. O. 1997. Tropical Forest Remnants: Ecology, management and conservation of fragmented communities. The University of Chicago Press, Chicago, USA, 616pp.

Ludwig, J. A.; Reynolds, J. F. 1988. Statistical Ecology: A primer on methods and computing. Wiley-Interscience Publication, New York, USA, 337pp.

Marques-Aguiar, S. P. 1994. A systematic review of the large species of Artibeus Leach, 1821 (Mammalia: Chiroptera), with some phylogenetic inferences. Boletim do Museu Paraense Emílio Goeldi Série Zoologia, 10 (1): 3-83.

Medellín, R. A.; Equihua, M.; Amin, M. A. 2000. Bat diversity and abundance as indicators of disturbance in Neotropical rainforest. Conservation Biology, 14 (6): 1666-1675.

Meneses-Filho, L. C. L.; Ferraz, P. A.; Pinha, J. F. M.; Ferreira, L. A.; Brilhante, N. A. 1995. Comportamento de 24 espécies arbóreas tropicais madeireiras introduzidas no Parque Zoobotânico de Rio Branco-Acre. v 1. Edufac, Rio Branco, Brasil, 135pp.

Menezes, L. F.; Duarte, A. C.; Novaes, R. L. M.; Façanha, A. C.; Peracchi, A. L.; Costa, L. M.; Fernandes, A. F. P. D.; Esbérard, C. E. L. 2008. Deslocamento de Artibeus lituratus (Olfers, 1818) (Mammalia, Chiroptera) entre ilha e continente no Estado do Rio de Janeiro, Brasil. Biota Neotropica, 8 (2): 243-245.

Nogueira, M. R.; Pol, A.; Peracchi, A. L. 1999. New records of bats from Brazil with a list of additional species for the chiropteran fauna of the state of Acre, western Amazon basin. Mammalia, 63 (3): 363-368.

Pedro, W. A.; Geraldes, M. P.; Lopez, G. G.; Alho, C. J. R. 1995. Fragmentação de habitat e a estrutura de uma taxocenose de morcegos em São Paulo (Brasil). Chiroptera Neotropical, 1 (1): 4-6.

Reis, N. R. 1984. Estrutura de comunidades de morcegos na região de Manaus, Amazonas. Revista Brasileira de Biologia, 44 (3): 247-254.

Reis, N. R.; Muller, M. F. 1995. Bat diversity of forests and open areas in a subtropical region of South Brazil. Ecologia Austral, 5: 31-36.
Reis, N. R.; Barbieri, M. L. S.; Lima, I. P.; Peracchi, A. L. 2003. O que é melhor para manter a riqueza de espécies de morcegos (Mammalia, Chiroptera): Um fragmento florestal grande ou vários fragmentos de pequeno tamanho? Revista Brasileira de Zoologia, 20 (2): $225-230$

Reis, N. R.; Peracchi, A. L.; Sekiama, M. L.; Lima, I. P. 2000. Diversidade de morcegos (Chiroptera, Mammalia) em fragmentos florestais no estado do Paraná, Brasil. Revista Brasileira de Zoologia, 17 (3): 697-704.

Sampaio, E. M.; Kalko, E. K. V.; Bernard, E.; Rodríguez-Herrera, B., Handley, C. O. 2003. A biodiversity assessment of bats (Chiroptera) in a tropical lowland rainforest to central Amazonia, including methodological and conservation considerations. Studies on Neotropical Fauna and Environment, 38 (1): 17-31.

Santos, A. J. 2003. Estimativas de riqueza em espécies. In: Cullen Jr., L.; Rudran, R. \& Valladares-Padua (Orgs). Métodos de Estudos em Biologia da Conservação e Manejo da Vida Silvestre. Editora UFPR, Curitiba, Brasil, p.19-41.

Silveira, M. 2005. Floresta aberta com bambu no sudoeste da Amazônia: Padrões e processos em múltiplas escalas. Edufac, Rio Branco, Brasil, 151pp.

Straube, F. C.; Bianconi, G. V. 2002. Sobre a grandeza e a unidade utilizada para estimar esforço de captura com utilização de redesde-neblina. Chiroptera Neotropical, 8 (1-2): 150-152.

Taddei, V. A.; Rezende, I. M.; Camora, D. 1990. Notas sobre uma coleção de morcegos de Cruzeiro do Sul, Rio Juruá, Estado do Acre (Mammalia, Chiroptera). Boletim do Museu Paraense Emílio Goeldi Série Zoologia, 6 (1): 75-88.

Vizotto, L. D.; Taddei, V. A. 1973. Chave para determinação de quirópteros brasileiros. Revista da Faculdade de Filosofia Ciências e Letras São José do Rio Preto - Boletim de Ciências, 1: 1-72.

Willig, M. R.; Presley, S. J.; Bloch, C. P.; Hice, C. L.; Yanoviak, S. P.; Díaz, M. M.; Chauca, L. A.; Pacheco, V.; Weaver, S. C. 2007. Phyllostomid bats of lowland Amazonia: Effects of habitat alteration on abundance. Biotropica, 39 (6): 737-746.

Wilson, D. E.; Reeder, D. M. 2005. Mammal species of the world: A taxonomic and geographic reference. v1. $3^{\text {rd }}$ ed. The Johns Hopkins University Press, Baltimore, USA, 743pp. 